\title{
Witnessing Phenotypic and Molecular Evolution in the Fruit Fly
}

\author{
Caiti S. S. Heil • Mika J. Hunter • Juliet K. F. Noor • \\ Kathleen Miglia • Brenda Manzano-Winkler • \\ Shannon R. McDermott • Mohamed A. F. Noor
}

Published online: 12 September 2012

(C) Springer Science+Business Media, LLC 2012

\begin{abstract}
This multi-day exercise is designed for a college genetics and evolution laboratory to demonstrate concepts of inheritance and phenotypic and molecular evolution using a live model organism, Drosophila simulans. Students set up an experimental fruit fly population consisting of ten white-eyed flies and one red-eyed fly. Having red eyes is advantageous compared to having white eyes, allowing students to track the spread of this advantageous trait over several generations. Ultimately, the students perform polymerase chain reaction and gel electrophoresis at two neutral markers, one located in close proximity to the eye color locus and one located at the other end of the chromosome. Students observe that most flies have red eyes, and these red-eyed flies have lost variation at the near marker but maintained variation at the far marker hence observing a "selective sweep" and the "hitchhiking" of a nearby neutral
\end{abstract}

C. S. S. Heil $(\varangle) \cdot$ J. K. F. Noor $\cdot$ K. Miglia $\cdot$

B. Manzano-Winkler $\cdot$ S. R. McDermott $\cdot$ M. A. F. Noor

Department of Biology, Duke University,

Box 90338, Durham, NC 27708, USA

e-mail: css25@duke.edu

J. K. F. Noor

e-mail: jkfnoor@duke.edu

K. Miglia

e-mail: kathleen.miglia@duke.edu

B. Manzano-Winkler

e-mail: bw64@duke.edu

S. R. McDermott

e-mail: srmcderm@gmail.com

M. A. F. Noor

e-mail: noor@duke.edu

M. J. Hunter

Riverside High School,

3218 Rose of Sharon Rd,

Durham, NC 27712, USA

e-mail: mika.hunter@dpsnc.net variant. Students literally observe phenotypic and molecular evolution in their classroom!

Keywords Drosophila · Genetics · Evolution · Selective sweep $\cdot$ Hitchhiking

\section{Introduction}

When asked about their views on evolution, merely $15 \%$ of the general American public responded that human beings definitely developed over millions of years from less advanced forms of life (Newport 2012). In the same poll, 46\% of people responded that God created humans in their present form (Newport 2012). Perhaps surprisingly, students enrolled in universities poll very similarly. Thirty-five percent of students believe that humans were present at the time of the dinosaurs, and $42 \%$ of students do not believe that human beings, as we know them today, developed from earlier species of animals (National Science Board 2000, p. A-549). In our introductory evolution class at Duke University, 55\% of enrolled students in spring, 2011, said that "there are holes in the evidence for the theory of evolution." Continuing evolution education is therefore a priority identified by many science and educational associations (see Alters and Nelson 2002).

Most people think of evolutionary change as producing or exaggerating physical differences, but evolution can also be observed at the molecular level. For example, when an advantageous mutation occurs, natural selection can push that mutation to high frequency, dragging along nearby neutral variants (Smith and Haigh 1974). Essentially, with the spread of an advantageous mutation over multiple generations (a "selective sweep"), the particular allele at nearby genes that was associated with the original mutation also spreads. The associated allele at these nearby genes "hitchhikes" alongside the 
spreading mutation and thereby decreases genetic variation in that region. However, alleles at genes far away from the original mutation fail to hitchhike and show more typical levels of genetic variation (see Fig. 1). The reason for this difference is linkage: recombination separates alleles if they are far away on a chromosome but cannot do so if the alleles are very close together in the genome. This is the same concept used in constructing linkage maps or genetic mapping of diseases.

Currently, most laboratory exercises and demonstrations focusing on evolution are limited to computer models and simulations. While useful for illustrating concepts, students tend to prefer activities with a greater "hands-on" component, especially involving live organisms.

The exercise presented here leverages the genetic model organism Drosophila simulans and allows students to witness evolution in their own classroom at the phenotypic and molecular levels. This laboratory exercise is an extension of a K-12 laboratory exercise (Heil et al. 2013) and is intended for college level introduction to biology and/or evolution courses. Briefly, students set up a population of fruit flies consisting of five white-eyed females, five white-eyed males, and one redeyed male. White eye color is a mutant phenotype, caused by a mutation in a gene in the pigment pathway, discovered in 1910 by the father of modern genetics, Thomas Hunt Morgan (Green 2010; Morgan 1910). The "white" mutation is located on the X chromosome and results in lack of pigment, poor eyesight, and lower fitness (Jones and Probert 1980; Reed and Reed 1950). The addition of the red-eyed male simulates the introduction of an advantageous mutation in an ancestrally white-eyed population. Over multiple generations, students witness the spread of the red eye phenotype, and they later genotype their flies at two variable genetic markers. One marker is located in close proximity to the eye color gene and one marker is located on the other end of the chromosome. Students observe that red-eyed flies all possess the same variant at the proximate ("Near") marker but possess either variant at the far-away ("Far") marker. White-eyed flies possess either variant at both markers. Hence, students witness the selective sweep of the advantageous red eye phenotype, the hitchhiking of a nearby neutral variant (Fig. 2), and the lack of hitchhiking at a far-away neutral variant. Sample associated PowerPoint lectures, an Appendix with detailed instructions, and student handouts are available for download at http://sites.google.com/ site/noorlabduke/fly-evolution-advanced, and educators are free to modify them for their individual use.

\section{Description of Activities}

\section{Learning Objectives}

Between the laboratory exercise and the associated lectures, the students will:

- Set up control white-eyed fruit fly populations and experimental ones with a "mutant" red-eyed male added

- Directly observe greater mating success of the red-eyed male
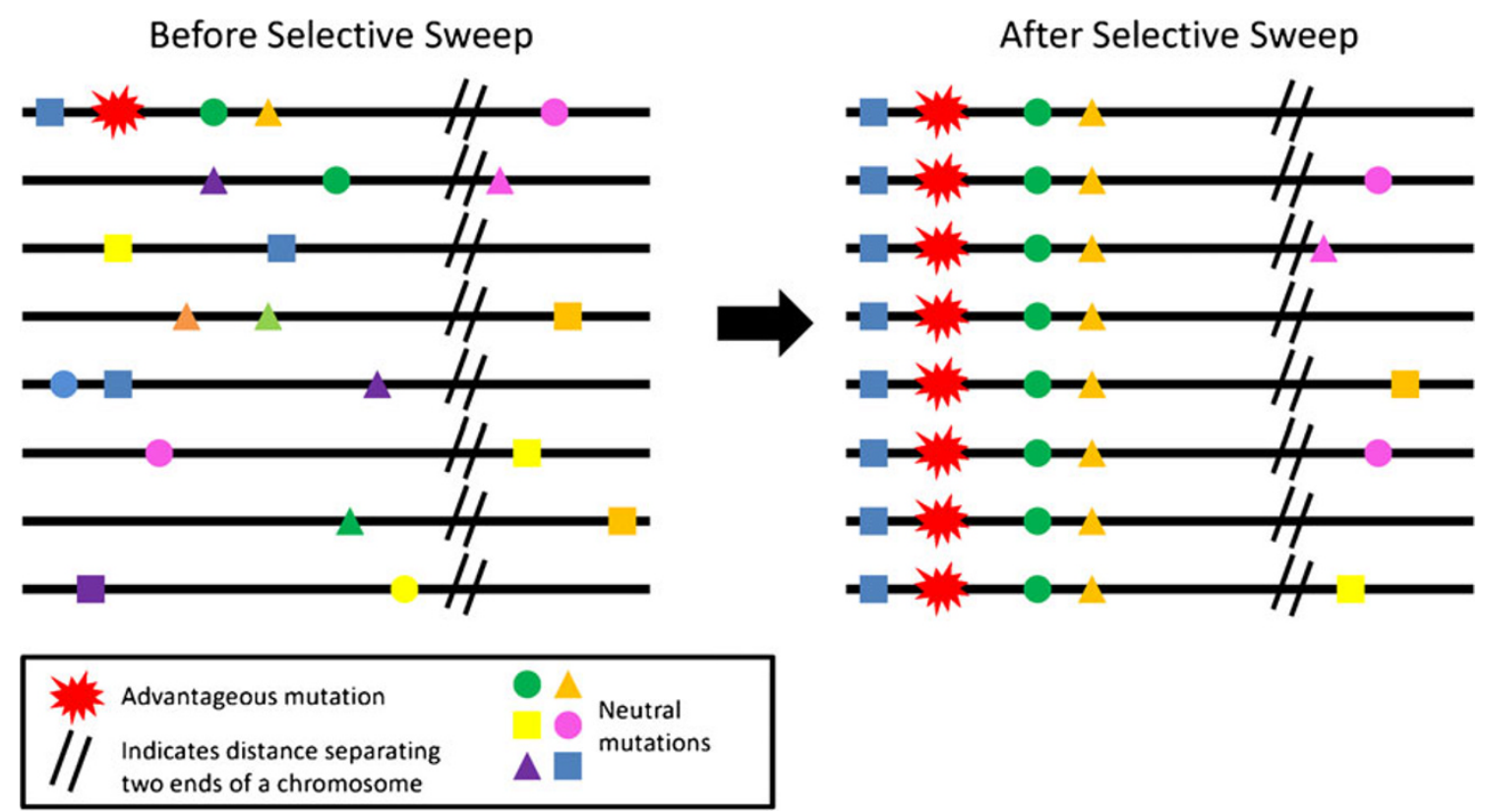

Fig. 1 When an advantageous mutation occurs, it rises to high frequency, dragging along nearby neutral variants. Eventually, every individual in the population has the same mutation and the nearby neutral variants that "hitchhiked" with it. All previous diversity is lost. Conversely, neutral variants located far away from the advantageous mutation do not hitchhike because recombination can break apart the association and therefore maintain variation 

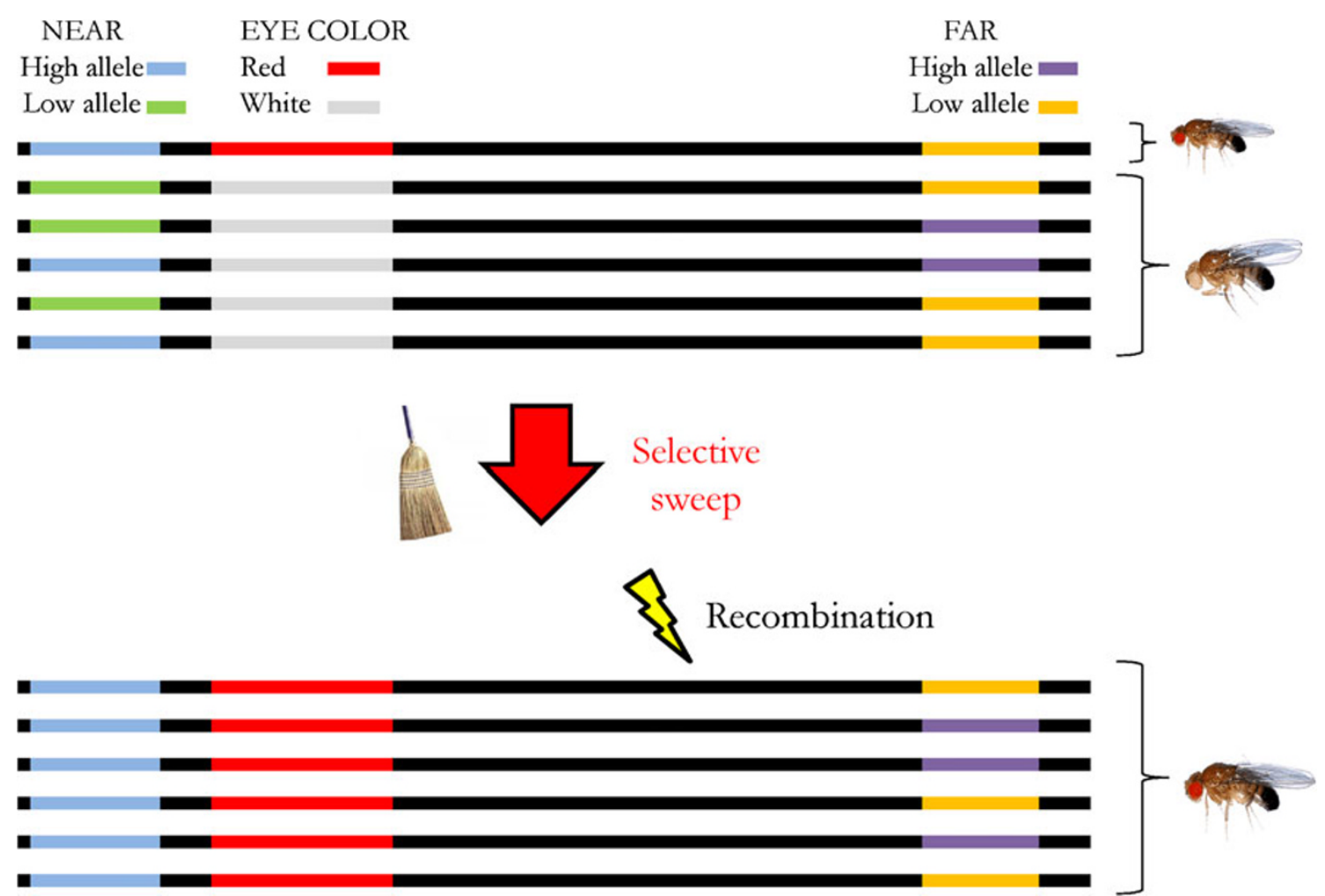

Fig. 2 The initial population consists of white-eyed flies that carry two alleles at the Near marker (green or blue), two alleles at the Far marker (purple or yellow), and white eye allele at the eye color gene. A redeyed fly is introduced that carries the blue allele at Near, yellow at Far, and red at the eye color gene. The red eye allele is advantageous and

- Identify that, as a result of the red-eyed males greater mating success, the red eye variant (allele) is more abundant in the next generations: a personally observed case of "evolution by natural selection"

- Understand $\mathrm{X}$-linkage, in part by seeing that none of the $F_{1}$ offspring males have red eyes even though most of the females do

- Learn about and apply polymerase chain reaction (PCR) and gel electrophoresis for the purpose of understanding an evolutionary process

- Learn about physical linkage of segments on the same chromosome

- Demonstrate the molecular evolution concept of "hitchhiking" of alleles at other genes along with the spread of an advantageous mutation

Materials

- D. simulans w138a (white), UC San Diego Drosophila Species Stock Center, stock number 14021-0251.138a

- D. simulans w195 (white), UC San Diego Drosophila Species Stock Center, stock number 14021-0251.195 sweeps to high frequency in the population. The blue allele at Near is located close to the advantageous eye color and hitchhikes with the red eye color. The Far allele is located 22 million base pairs down at the other end of the chromosome. Recombination breaks down linkage between eye color and Far, so both yellow and purple alleles persist

- D. simulans C167.4 (wild type), UC San Diego Drosophila Species Stock Center, stock number 14021-0251.199

- Carolina Biological Supply: Drosophila Kit (FlyNap ${ }^{\circledR}$, 72 vials, 72 plugs, media, labels, transfer cards): 173052

- (Optional but strongly preferred) $\mathrm{CO}_{2}$ anesthetization facilities for Drosophila

- "Squishing buffer" and proteinase-K for DNA isolation (protocol in Appendix)

- Equipment and reagents for polymerase chain reaction including oligonucleotides for "Near" and "Far" markers in the Appendix

- Equipment and reagents for gel electrophoresis

- Magnifying glasses

- Sharpie markers

- PowerPoint lectures (available online) and projector (optional)

Material Notes Because of the availability of multiple wholegenome sequences (Begun et al. 2007) that were leveraged in its design, this laboratory exercise uses the species $D$. simulans rather than its sister species Drosophila melanogaster. These stocks can be ordered from the UCSD Stock Center, and the crossing scheme, which must be started approximately 
eightweeks in advance of the first class exercise, can be found in the supplementary materials. Oligonucleotide primers for PCR of the near and far markers must also be ordered commercially (we use Integrated DNA Technologies). Other materials can be purchased from a science supply company, such as Carolina Biological Supply. Alternatively, many of these materials may be already available at your university for use in other (especially molecular biology) laboratory activities. This lab requires additional resources, preparation time, and activity time that may not be available in most K-12 schools and some colleges. However, it has been implemented successfully both at Duke University for the introductory course (sophomore level) in genetics and evolution and a public high school advanced placement biology class with assistance and volunteers from a Duke University research laboratory. We recommend colleges lacking molecular resources to consider implementing only the portion of the lab outlined in Heil et al. (2013).

This laboratory exercise is designed to take place on multiple days over several months. If lab time is limited, several of these steps can be omitted or conducted by the instructor or preparatory staff. For example, day two can be omitted without much loss of content. Furthermore, if the class is already familiar with PCR, the PCR and gel electrophoresis can be done outside of lab and gel pictures can be returned to students the next week for analysis.

\section{Day One}

Setting up Your Population (Time 20 Minutes) Students are provided two vials: one containing five white-eyed females and one containing five white-eyed males and one red-eyed male. Students are asked to observe any differences in the phenotype among the flies. Once the difference in eye color is identified, students should be asked to make a prediction about what will happen to the frequency of the red eye phenotype if it is advantageous or disadvantageous. Students then carefully transfer the contents of one vial into the other, then place the vial sideways on a white piece of paper and observe. Often, students will witness matings, almost always between the red-eyed male and a whiteeyed female. Videos of Drosophila courtship behavior can be easily found online and make good supplementary presentations.

\section{Day Two}

Demonstration of X-linkage (Optional, Time 20 Minutes) After one generation (two to three weeks), some of the offspring from the cross set up on day one can be returned to the students. Students sort them into one of four assigned categories: white-eyed females, white-eyed males, red-eyed males, and red-eyed females. Because the eye color gene is sex-linked, no males will have red eyes (Fig. 3). This is a clear demonstration of sex-linked inheritance. For a more complete description of days one and two, see Heil et al. (2013) and online materials.

\section{Day Three}

Demonstration of Evolution by Natural Selection (Time 15 Minutes) After three to four generations (approximately three months), the descendants of the original flies are returned to the students to observe the extent of change in abundance of the red-eye variant. Most of the flies of both sexes will be red; therefore, students can conclude that the red eye variant was advantageous. If a student's vial has a frequency of red-eyed flies greater than $1 / 11$, evolution by natural selection has proceeded in this population: evolution is evident through the change in frequency and natural selection is evident because it occurs in a predictable manner (increase in abundance of the red-eye variant).

DNA Isolation (Time 45 Minutes) The students will place seven male flies (three to four of each eye color) into eight labeled 0.2-milliliter microcentrifuge tubes (leaving one for a negative control), then squish them with a separate pipette tip for each fly in the presence of squishing buffer with proteinase-K (Gloor and Engels 1992). The DNA preparation can be incubated at 37 degrees Celsius for at least 30 minutes in a thermal cycler or alternatively can be left at room temperature for the same duration, and the proteinase can be inactivated by a two-minute incubation at

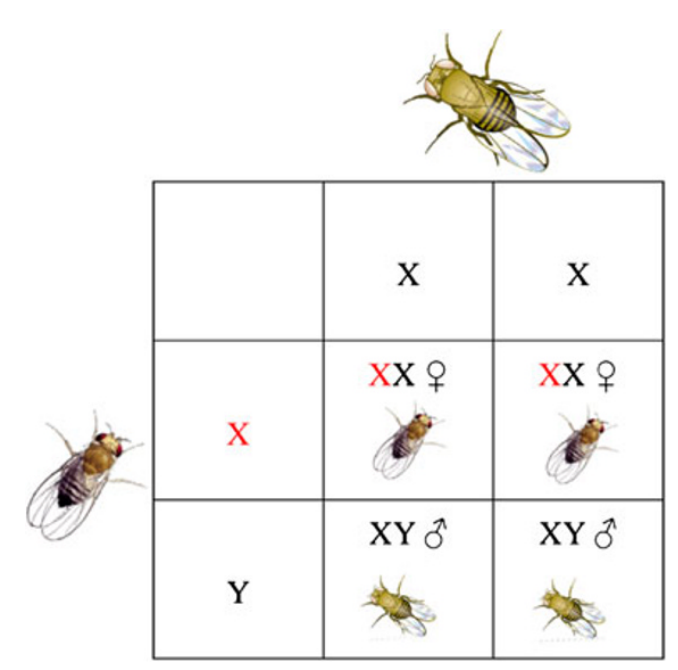

Fig. 3 The eye color gene is located on the X chromosome (one of the sex-determining chromosomes of Drosophila). White eye color is recessive. When a red-eyed male mates with white-eyed females, their daughters will have red eyes, but their sons will have white eyes. Therefore, there can be no red-eyed males in the first generation, but they will appear in the second and later generations. From Heil et al. (2013) 
95 degrees Celsius. This will extract and stabilize the fly DNA for the PCR.

Polymerase Chain Reaction (Time 15-30 Minutes Preparation, Plus $\sim 1.5$ Hours Thermal Cycler) This laboratory can be done with DNA isolation if the laboratory period is 2.5 hours or longer or can be completed by laboratory prep staff outside of lab. Students use their DNA preparations from day three and amplify two target DNA fragments via PCR: one near the eye color gene and another at the other end of the X chromosome. The products of the PCR will be visualized using gel electrophoresis and analyzed in day four.

\section{Day Four}

Gel Electrophoresis (Time 60+ Minutes) Students begin by practicing loading a gel, then load their 16 amplified samples from day three (eight samples from "Near" marker PCR and eight samples from "Far" marker PCR) in a previously prepared 1.8\% lithium borate agarose gel with Sybr Safe. The gel is run at 300 volts for 45 minutes. Photographs of the electrophoresed, amplified DNA will then be taken and given to the students.

Data Analysis: Concepts of Linkage and hitchhiking (Time 30 Minutes) Data analysis can be completed on another day if time or equipment does not allow for the gel electrophoresis to finish in one class period. Students will analyze their gel images and score the alleles observed at target markers near to vs. far from the eye color gene. They will observe that all (or nearly all) red-eyed males have the same allele at the "Near" marker, whereas white-eyed males have either of two alleles (see Figs. 2 and 4). Students then infer what happens over the next few generations. Eventually, the entire population will have the red eye color variant. At that time, no genetic variation will remain at the "Near" marker. This loss of variation in red-eyed males associated with hitchhiking is called a selective sweep (Berry et al. 1991). In contrast, the "Far" marker bears two alleles among individuals in both the original white-eyed males and still in the new red-eyed males (see Figs. 2 and 4). These concepts are discussed extensively in studies of and classes on molecular evolution and genome evolution, and the students will have had the unique opportunity to witness the processes in action in this exercise.

\section{Assessments}

While we do not have pre- and post-laboratory assessments of learning objectives and student learning, we do have student evaluations of this exercise by itself and relative to other labs conducted over the course of a semester. Roughly 1,000 students have completed this exercise to date, and with the available data, this series of lab exercises was ranked as the most interesting lab of eight labs conducted in the semester. When asked questions regarding to what

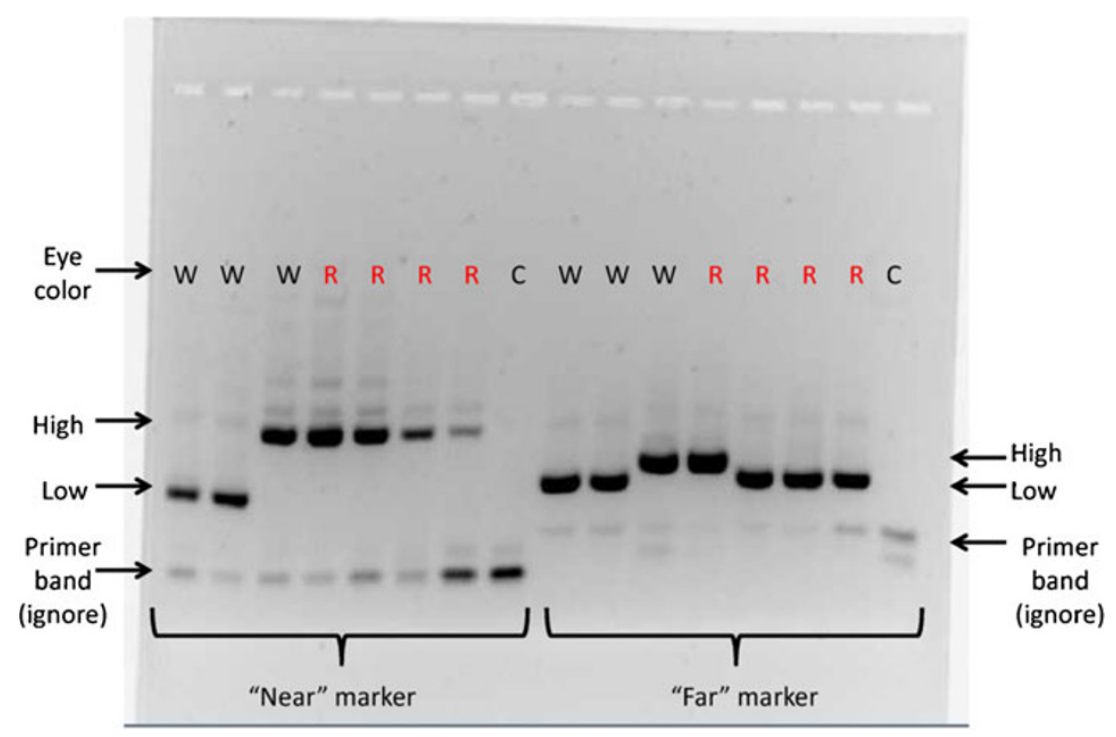

Fig. 4 A sample student's gel. At the top of the gel, the eye color of the sample is noted as $W$ (white-eyed), $R$ (red-eyed), or $C$ (control, no DNA present in sample). On the left of the gel are the results for the Near marker, the marker located in close proximity to the eye color gene. The original red-eyed male had a high band at the Near marker. One can see that all red-eyed flies have the high band at the Near marker, illustrating the concept of a nearby neutral variant hitchhiking with an advantageous mutation (the red-eye color). On the right side of the gel are the results for the Far marker, which is located on the other end of the chromosome from the eye color gene. The Far marker clearly has less size differential between the high and low bands, so instruct students to us caution in interpreting results. The original red-eyed male had a low band at the Far marker, but one can see that red-eyed flies in the current sample have both high and low bands, illustrating the concept that recombination breaks up the association between the eye color gene and neutral variants located far away on the chromosome 
extent the laboratory helped the students to understand the course material better and to what degree the laboratory increased their interest in the topics presented, results were very similar for both questions, with about $67 \%$ of students ranking the labs as a four or five (scale of one to five, with one being "not so much" and five being "very much," $1=$ $2 \%, 2=8 \%, 3=23 \%, 4=42 \%, 5=25 \%$ ). Eighty-two percent of over 200 students from the most recent iteration of the lab indicated they enjoyed the labs, and $40 \%$ responded they highly or very highly enjoyed them (scale of one to five with one being not so much, three being "moderately," and five being "very highly," of students, $1=2 \%, 2=14 \%, 3=42 \%$, $4=33 \%, 5=7 \%$ ).

\section{Conclusions}

In this exercise, students observe the central components of Darwin's theory of evolution by natural selection: (1) individuals in a population are variable (red and white eyes), (2) this variation is inherited from parent to offspring (witnessed in sex-linked inheritance), and (3) inherited variations that help individuals to survive and reproduce better than others will become more abundant in the population (the white eye phenotype being disadvantageous and the spread of the red eye phenotype). Students can observe that the composition of the population changes from one generation to the next in a predictable manner: it changes from a mostly white-eyed population of flies to a majority red-eyed population (Heil et al. 2013).

Furthermore, students have the opportunity to witness molecular evolutionary change. The locus near the spreading advantageous eye color mutation loses one of the two alleles initially present, representing a loss of variation near the site of the mutation. In contrast, at the "Far" marker, both alleles present in the original population remain at the end of the experiment. Recombination events in between the eye color locus and the "Far" marker allowed the "Far" marker to become disassociated with eye color (Figs. 2 and 4).

Researchers have used these patterns of decreased variation following a selective sweep to detect and map genetic adaptation in many organisms, including humans. For example, recent studies looking at regions with decreased genetic variation in human genomes identified mutations in the human lactase gene that occurred within the last 10,000 years that allow us to consume dairy as adults (Schaffner and Sabeti 2008). Looking at patterns of variation across human populations helped identify substitutions in Tibetans that appear to be adaptive for living at high altitudes, like the EPAS1 locus, which is involved in the body's response to hypoxia or decreased oxygen supply (Simonson et al. 2010; Yi et al. 2010).
Through these exercises, students will utilize a genetic model organism to personally observe a case of evolution by natural selection and apply PCR and gel electrophoresis for the purpose of understanding an evolutionary process. In the end, students will have a greater understanding of inheritance, selective sweeps and hitchhiking, and will have literally observed evolution by natural selection within their classroom.

Acknowledgments Funding for this project came from a National Science Foundation's Research Experience for Teachers awarded to Mohamed Noor and Mika Hunter. We would like to thank teachers and students at Riverside High School in Durham for trying out this exercise and the preparatory staff for the Genetics and Evolution laboratories at Duke University for help incorporating the lab into the Duke University biology curriculum.

\section{References}

Alters BJ, Nelson CE. Perspective: teaching evolution in higher education. Evolution. 2002;56(10):1891-901.

Begun DJ, Holloway AK, Stevens K, Hillier LW, Poh YP, Hahn MW, et al. Population genomics: whole-genome analysis of polymorphism and divergence in Drosophila simulans. Plos Biology. 2007;5 (11):2534-59.

Berry AJ, Ajioka JW, Kreitman M. Lack of polymorphism on the drosophila 4th chromosome resulting from selection. Genetics. 1991;129(4):1111-7.

Gloor G, Engels W. Single-fly DNA preps for PCR. Drosoph Inf Serv. 1992;71:148-9.

Green MM. 2010: a century of Drosophila genetics through the prism of the white gene. Genetics. 2010;184(1):3-7.

Heil CSS, Manzano-Winkler B, Hunter MJ, Noor JKF, Noor MAF. Witnessing evolution first-hand: a K-12 laboratory exercise in genetics and evolution using Drosophila. Am Biol Teach. 2013; in press

Jones JS, Probert RF. Habitat selection maintains a deleterious allele in a heterogeneous environment. Nature. 1980;287(5783):632-3.

Morgan TH. Sex limited inheritance in Drosophila. Science. 1910;32 (1):120-2.

National Science Board. Science and engineering indicators 2000 (NSB00-1). Washington, DC: U.S. Government Printing Office; 2000.

Newport, F. In U.S., 46\% Hold Creationist View of Human Origins. 2012. Available online at http://www.gallup.com/poll/155003/HoldCreationist-View-Human-Origins.aspx. Accessed 01 June 2012.

Reed SC, Reed EW. Natural selection in laboratory populations of Drosophila. 2. Competition between a white-eye gene and its wild type allele. Evolution. 1950;4(1):34-42.

Schaffner SF, Sabeti P. Evolutionary adaptation in the human lineage. Nature Education. 2008;1(1).

Simonson TS, Yang YZ, Huff CD, Yun HX, Qin G, Witherspoon DJ, et al. Genetic evidence for high-altitude adaptation in Tibet. Science. 2010;329(5987):72-5.

Smith JM, Haigh J. Hitch-hiking effect of a favorable gene. Genet Res. 1974;23(1):23-35.

Yi X, Liang Y, Huerta-Sanchez E, Jin X, Cuo ZXP, Pool JE, et al. Sequencing of 50 human exomes reveals adaptation to high altitude. Science. 2010;329(5987):75-8. 